\title{
On $\mathscr{S}^{\star}$-closed sets and some low separation axioms in ideal topological spaces
}

\author{
CHAWALIT BOONPOK \\ Mathematics and Applied Mathematics Research Unit \\ Department of Mathematics, Faculty of Science, Mahasarakham University \\ Maha Sarakham, 44150 \\ THAILAND
}

\begin{abstract}
This paper deals with the concepts of $\mathscr{S}^{\star}$-closed sets and $\mathscr{S}^{\star}$-open sets which are defined by utilizing thenotionof $\Lambda_{I}$-sets.Somecharacterizationsofsemi- $\mathscr{I}-R_{0}$ idealtopologicalspacesareinvestigated.

Key-Words: $\Lambda_{I}^{s}$-set, $\mathscr{S}^{\star}$-closed set, $\mathscr{S}^{\star}$-open set, semi- $\mathscr{I}$ - $R_{0}$ space

Received: November 24, 2019. Revised: May 2, 2020. Accepted: May 25, 2020. Published: June 23, 2020.
\end{abstract}

\section{Introduction}

The concepts of closed sets and some low separation axioms are fundamental with respect to the investigation of general topological spaces. In 1956, Maki [17] introduced the concept of $\Lambda$-sets in topological spaces as the sets that coincide with their kernel. The kernel of a set $A$ is the intersection of all open superset $A$. Arenas et al. [1] introduced and investigated the concept of $\lambda$-closed sets by involving $\Lambda$-sets and closed sets. Caldas and Jafari [2] introduced the concept of $\lambda$-closure of a set by utilizing the notion of $\lambda$-open sets defined in [1]. In [3], the present authors introduced and studied two new low separation axioms called $\lambda$ $R_{0}$ and $\lambda-R_{1}$ by utilizing the notions of $\lambda$-open sets and $\lambda$-closure operator. Keskin and Noiri [15] investigated some properties of $\gamma-R_{0}$ and $\gamma-R_{1}$ spaces due to Ekici $[8,9]$. Veličko [19] introduced and investigated the concepts of $\delta$-open sets, $\delta$-closure operator and $\delta$-closed sets. Georgiou et al. [10] by considering the notion of $\delta$-closed sets, introduced and investigated $\Lambda_{\delta}$-sets, $(\Lambda, \delta)$-closed sets, $(\Lambda, \delta)$-open sets and $(\Lambda, \delta)$-closure operator. Caldas et al. [5] introduced and investigated some new low separation axioms by using the notions of $(\Lambda, \delta)$-open sets and $(\Lambda, \delta)$-closure operator. In [4], the present authors introduced and studied two new weak separation axioms called $\Lambda_{\theta}-R_{0}$ and $\Lambda_{\theta}-R_{1}$ spaces by using the notions of $(\Lambda, \theta)$-open sets and $(\Lambda, \theta)$-closure operator.

In 2002, Hatir and Noiri [13] have introduced the notions of $\alpha-\mathscr{I}$-open sets, semi- $\mathscr{I}$-open sets and $\beta$ $\mathscr{I}$-open sets in topological spaces via ideals and obtained new decompositions of continuity. Later, in [12], the same authors investigated the further properties of semi- $\mathscr{I}$-open sets and semi- $\mathscr{I}$-continuous functions introduced in [13]. Recently, Sanabria et al. [18] introduced and studied the notions of $\Lambda_{I}^{s}$-sets, $\Lambda_{I}^{s}$-closed sets and $\mathscr{I}$-generalized semi-closed sets by using semi- $\mathscr{I}$-open sets in ideal topological spaces.

The purpose of the present paper is to introduce the notions of $\mathscr{S}^{\star}$-closed and $\mathscr{S}^{\star}$-open sets. Moreover, several interesting fundamental properties of $\mathscr{S}^{\star}$-closed and $\mathscr{S}^{\star}$-open sets are investigated. Additionally, we introduce the notion of semi- $\mathscr{I}-R_{0}$ ideal topological spaces and investigate some characterizations of such spaces.

\section{Preliminaries}

Throughout the present paper, spaces $(X, \tau)$ and $(Y, \sigma)$ (or simply $X$ and $Y$ ) always mean topological spaces on which no separation axioms are assumed unless explicitly stated. In a topological space $(X, \tau)$, the closure and the interior of any subset $A$ of $X$ will denoted by $\mathrm{Cl}(A)$ and $\operatorname{Int}(A)$, respectively. An ideal $\mathscr{I}$ on a topological space $(X, \tau)$ is a nonempty collection of subsets of $X$ satisfying the following properties: (1) $A \in \mathscr{I}$ and $B \subseteq A$ implies $B \in \mathscr{I}$; (2) $A \in \mathscr{I}$ and $B \in \mathscr{I}$ implies $A \cup B \in \mathscr{I}$. A topological space $(X, \tau)$ with an ideal $\mathscr{I}$ on $X$ is called an ideal topological space and is denoted by $(X, \tau, \mathscr{I})$. For an ideal topological space $(X, \tau, \mathscr{I})$ and a subset $A$ of $X, A^{\star}(\mathscr{I})$ is defined as follows: $A^{\star}(\mathscr{I})=\{x \in X: U \cap A \notin \mathscr{I}$ for every open neighbourhood $U$ of $x\}$. In case there is no chance for confusion, $A^{\star}(\mathscr{I})$ is simply written as $A^{\star}$. In [16], $A^{\star}$ is called the local function of $A$ with respect to $\mathscr{I}$ and $\tau$ and $\mathrm{Cl}^{\star}(A)=A^{\star} \cup A$ defines a Kuratowski closure operator for a topology $\tau^{\star}(\mathscr{I})$. For every ideal topological space $(X, \tau, \mathscr{I})$, there exists a topology $\tau^{\star}(\mathscr{I})$ finer than $\tau$, generated by 
$\mathscr{B}(\tau, \mathscr{I})=\left\{U-I_{0} \mid U \in \tau\right.$ and $\left.I_{0} \in \mathscr{I}\right\}$, but in general $\mathscr{B}(\tau, \mathscr{I})$ is not always a topology [14]. A subset $A$ of an ideal topological space $(X, \tau, \mathscr{I})$ is said to be $\star$-closed [14] if $A^{\star} \subseteq A$. The complement of a $\star$-closed set is said to be $\star$-open. The interior of a subset $A$ in $\left(X, \tau^{\star}(\mathscr{I})\right)$ is denoted by $\operatorname{Int}^{\star}(A)$.

Definition 1. [13] A subset $A$ of an ideal topological space $(X, \tau, \mathscr{I})$ is said to be semi-I $\mathscr{I}$-open if

$$
A \subseteq C l^{\star}(\operatorname{Int}(A)) .
$$

The complement of a semi- $\mathscr{I}$-open set is called semi$\mathscr{I}$-closed.

By $s \mathscr{I} O(X, \tau)$ (resp. $s \mathscr{I} C(X, \tau)$ ), we denote the family of all semi- $\mathscr{I}$-open (resp. semi- $\mathscr{I}$-closed) sets of an ideal topological space $(X, \tau, \mathscr{I})$. For a subset $A$ of an ideal topological space $(X, \tau, \mathscr{I})$, the intersection of all semi- $\mathscr{I}$-open sets containing $A$ is called semi- $\mathscr{I}$-closure [7] of $A$ and is denoted by $s \mathrm{Cl}_{\mathscr{I}}(A)$. The semi- $\mathscr{I}$-interior [7] of $A$, denoted by $s \operatorname{Int}_{\mathscr{I}}(A)$, is defined by the union of all semi- $\mathscr{I}$-open sets of $X$ contained in $A$.

Lemma 2. [12] Let $(X, \tau, \mathscr{I})$ be an ideal topological space and $A, B$ subsets of $X$.

(1) If $U_{\gamma} \in s \mathscr{I} O(X, \tau)$ for each $\gamma \in \Gamma$, then $\cup_{\gamma \in \Gamma} U_{\gamma} \in s \mathscr{I} O(X, \tau)$.

(2) If $A \in s \mathscr{I} O(X, \tau)$ and $B \in \tau$, then

$$
A \cap B \in s \mathscr{I} O(X, \tau) .
$$

Definition 3. [18] Let $A$ be a subset of an ideal topological space $(X, \tau, \mathscr{I})$. A subset $\Lambda_{I}^{s}(A)$ is defined as follows: $\Lambda_{I}^{s}(A)=\cap\{U \mid A \subseteq U, U \in s \mathscr{I} O(X, \tau)\}$.

Lemma 4. [18] Let $A, B$ and $\left\{B_{\gamma}: \gamma \in \Gamma\right\}$ be subsets of an ideal topological space $(X, \tau, \mathscr{I})$. Then

(1) $A \subseteq \Lambda_{I}^{s}(A)$.

(2) $\Lambda_{I}^{s}\left(\Lambda_{I}^{s}(A)\right)=\Lambda_{I}^{s}(A)$.

(3) If $A \subseteq B$, then $\Lambda_{I}^{s}(A) \subseteq \Lambda_{I}^{s}(B)$.

(4) If $A \in s \mathscr{I} O(X, \tau)$, then $A=\Lambda_{I}^{s}(A)$.

(5) $\Lambda_{I}^{s}\left(\underset{\gamma \in \Gamma}{\cup} B_{\gamma}\right)=\underset{\gamma \in \Gamma}{\cup} \Lambda_{I}^{s}\left(B_{\gamma}\right)$.

(6) $\Lambda_{I}^{s}\left(\bigcap_{\gamma \in \Gamma} B_{\gamma}\right) \subseteq \underset{\gamma \in \Gamma}{\cap} \Lambda_{I}^{s}\left(B_{\gamma}\right)$.

Definition 5. [18] Let $(X, \tau, \mathscr{I})$ be an ideal topological space. A subset $A$ of $X$ is said to be $\Lambda_{I}^{s}$-set if $A=\Lambda_{I}^{s}(A)$. The family of all $\Lambda_{I}^{s}$-sets of $(X, \tau, \mathscr{I})$ is denoted by $\Lambda_{I}^{s}(X)$ (or simply $\left.\Lambda_{I}^{s}\right)$.
Lemma 6. [18] Let $(X, \tau, \mathscr{I})$ be an ideal topological space. Then

(1) $\emptyset$ and $X$ are $\Lambda_{I}^{s}$-sets.

(2) For every subset $A$ of $X, \Lambda_{I}^{s}(A)$ is a $\Lambda_{I}^{s}$-set.

(3) If $A \in s \mathscr{I} O(X, \tau)$, then $A$ is a $\Lambda_{I}^{s}$-set.

(4) Every union of $\Lambda_{I}^{s}$-sets is a $\Lambda_{I}^{s}$-set.

(5) Every intersection of $\Lambda_{I}^{s}$-sets is a $\Lambda_{I}^{s}$-set.

Proposition 7. Let $(X, \tau, \mathscr{I})$ be an ideal topological space. Then $\Lambda_{I}^{s}(X)=\Lambda_{\Lambda_{I}^{s}}(X)$.

Proof. By Lemma 6, s $\mathscr{I} O(X, \tau) \subseteq \Lambda_{I}^{s}(X)$. Let $A$ be any subset of $X$. Then, we have

$$
\begin{aligned}
\Lambda_{\Lambda_{I}^{s}}(A) & =\cap\left\{U \mid A \subseteq U, U \in \Lambda_{I}^{s}(X)\right\} \\
& \subseteq \cap\{U \mid A \subseteq U, U \in s \mathscr{I} O(X, \tau)\} \\
& =\Lambda_{I}^{s}(A) .
\end{aligned}
$$

Therefore, $\Lambda_{\Lambda_{I}^{s}}(A) \subseteq \Lambda_{I}^{s}(A)$. Now, we suppose that $x \notin \Lambda_{\Lambda_{I}^{s}}(A)$. Then, there exists $U \in \Lambda_{I}^{s}(X)$ such that $A \subseteq U$ and $x \notin U$. Since $x \notin U$, there exists a semi$\mathscr{I}$-open set $V$ such that $U \subseteq V$ and $x \notin V$. Thus, $x \notin \Lambda_{I}^{s}(A)$. This shows that $\Lambda_{\Lambda_{I}^{s}}(A) \supseteq \Lambda_{I}^{s}(A)$ and hence $\Lambda_{I}^{s}(A)=\Lambda_{\Lambda_{I}^{s}}(A)$.

Definition 8. [18] An ideal topological space $(X, \tau, \mathscr{I})$ is said to be semi- $\mathscr{I}-T_{1}$ if for each pair of distinct points $x$ and $y$ of $X$, there exist semi- $\mathscr{I}$ open sets $U$ and $V$ of $X$ such that $y \in U, y \notin U$ and $y \in V, x \notin V$.

Lemma 9. [18] For an ideal topological space $(X, \tau, \mathscr{I})$, the following properties are equivalent:

(1) $(X, \tau, \mathscr{I})$ is semi- $\mathscr{I}-T_{1}$.

(2) For each $x \in X$, the singleton $\{x\}$ is a $\Lambda_{I}^{s}$-set.

(3) For each $x \in X$, the singleton $\{x\}$ is semi-Iclosed.

Proposition 10. An ideal topological space $(X, \tau, \mathscr{I})$ is semi- $\mathscr{I}-T_{1}$ if and only if the topological $\left(X, \Lambda_{I}^{s}(X)\right)$ is discrete.

Proof. Suppose that $(X, \tau, \mathscr{I})$ is semi- $\mathscr{I}-T_{1}$. Let $x \in X$, then by Lemma $9,\{x\}$ is a $\Lambda_{I}^{s}$-set and $\{x\}$ is open in $\left(X, \Lambda_{I}^{s}(X)\right)$. Thus, every subset of $X$ is open in $\left(X, \Lambda_{I}^{s}(X)\right)$ and hence $\left(X, \Lambda_{I}^{s}(X)\right)$ is discrete.

Conversely, suppose that a topological space $\left(X, \Lambda_{I}^{s}(X)\right)$ is discrete. For each $x \in X,\{x\}$ is open in $\left(X, \Lambda_{I}^{s}(X)\right)$ and hence $\{x\}$ is a $\Lambda_{I}^{s}$-set. Therefore, $(X, \tau, \mathscr{I})$ is semi- $\mathscr{I}-T_{1}$ by Lemma 9 . 


\section{$3 \mathscr{S}^{\star}$-closed sets in ideal topological spaces}

In this section, we introduce the notion of $\mathscr{S}^{\star}$-closed sets in ideal topological spaces. We also investigate some of their fundamental properties.

Definition 11. A subset $A$ of an ideal topological space $(X, \tau, \mathscr{I})$ is said to be $\mathscr{S}^{\star}$-closed if $A=T \cap C$, where $T$ is a $\Lambda_{I}^{s}$-set and $C$ is a semi- $\mathscr{I}$-closed set. The family of all $\mathscr{S}^{\star}$-closed sets in an ideal topological space $(X, \tau, \mathscr{I})$ is denoted by $\mathscr{S}^{\star} C(X)$.

Lemma 12. For a subset $A$ of an ideal topological space $(X, \tau, \mathscr{I})$, the following properties hold:

(1) $s \operatorname{Cl}_{\mathscr{I}}(A)=A \cup \operatorname{Int} t^{\star}(C l(A))$ [7].

(2) $\operatorname{sint}_{\mathscr{I}}(A)=A \cap C l^{\star}(\operatorname{Int}(A))$.

Theorem 13. For a subset $A$ of an ideal topological space $(X, \tau, \mathscr{I})$, the following properties are equivalent:

(1) A is $\mathscr{S}^{\star}$-closed.

(2) $A=T \cap s l_{\mathscr{I}}(A)$, where $T$ is a $\Lambda_{I}^{s}$-set.

(3) $A=\Lambda_{I}^{s}(A) \cap s C l_{\mathscr{I}}(A)$.

(4) $\operatorname{Int}^{\star}(C l(A)) \cap \Lambda_{I}^{s}(A) \subseteq A$.

Proof. (1) $\Rightarrow(2)$ : Let $A=T \cap C$, where $T$ is a $\Lambda_{I^{-}}^{s}$ set and $C$ is a semi- $\mathscr{I}$-closed set. Since $A \subseteq C$, we have $s \mathrm{Cl}_{\mathscr{I}}(A) \subseteq C$ and hence

$$
\begin{aligned}
A & =T \cap C \\
& \supseteq T \cap s \mathrm{Cl}_{\mathscr{I}}(A) \\
& \supseteq A .
\end{aligned}
$$

Consequently, we obtain $A=T \cap s \mathrm{Cl}_{\mathscr{I}}(A)$.

$(2) \Rightarrow(3)$ : Let $A=T \cap s \mathrm{Cl}_{\mathscr{I}}(A)$, where $T$ is a $\Lambda_{I}^{s}$-set. Since $A \subseteq T$, we have $\Lambda_{I}^{s}(A) \subseteq \Lambda_{I}^{s}(T)=T$ and hence

$$
\begin{aligned}
A & \subseteq \Lambda_{I}^{s}(A) \cap s \mathrm{Cl}_{\mathscr{I}}(A) \\
& \subseteq T \cap s \mathrm{Cl}_{\mathscr{I}}(A) \\
& =A .
\end{aligned}
$$

Thus, $A=\Lambda_{I}^{s}(A) \cap s \mathrm{Cl}_{\mathscr{I}}(A)$.

$(3) \Rightarrow(4)$ : Let $A=\Lambda_{I}^{s}(A) \cap s \mathrm{Cl}_{\mathscr{I}}(A)$. By (3) and Lemma 12(1), we have

$$
\begin{aligned}
A & =\Lambda_{I}^{s}(A) \cap\left[A \cup \operatorname{Int}^{\star}(\mathrm{Cl}(A))\right] \\
& =\left(\Lambda_{I}^{s}(A) \cap A\right) \cup\left[\Lambda_{I}^{s}(A) \cap \operatorname{Int}^{\star}(\operatorname{Cl}(A))\right] \\
& =A \cup\left[\Lambda_{I}^{s}(A) \cap \operatorname{Int}^{\star}(\mathrm{Cl}(A))\right]
\end{aligned}
$$

and hence $\operatorname{Int}^{\star}(\mathrm{Cl}(A)) \cap \Lambda_{I}^{s}(A) \subseteq A$.

$(4) \Rightarrow(1)$ : Let $\operatorname{Int}^{\star}(\operatorname{Cl}(A)) \cap \Lambda_{I}^{s}(A) \subseteq A$. Then $A \cup\left[\Lambda_{I}^{s}(A) \cap \operatorname{Int}^{\star}(\operatorname{Cl}(A))\right]=A$ and by Lemma 12(1),

$$
\begin{aligned}
A & =\left(A \cup \Lambda_{I}^{s}(A)\right) \cap\left[A \cup \operatorname{Int}^{\star}(\operatorname{Cl}(A))\right] \\
& =\Lambda_{I}^{s}(A) \cap s \mathrm{Cl}_{\mathscr{I}}(A) .
\end{aligned}
$$

This shows that $A$ is $\mathscr{S}^{\star}$-closed.

Definition 14. An ideal topological space $(X, \tau, \mathscr{I})$ is said to be semi-I $\mathscr{I}-T_{0}$ if for any distinct pair of points in $X$, there exists a semi- $\mathscr{I}$-open set containing one of the points but not the other.

It is obvious that every semi- $\mathscr{I}-T_{1}$ space is semi$\mathscr{I}-T_{0}$. However, the converse is not true as seen from the next example.

Example 15. Let $X=\{a, b\}$ with a topology

$$
\tau=\{\emptyset,\{a\}, X\}
$$

and an ideal $\mathscr{I}=\{\emptyset,\{b\}\}$. Then $(X, \tau, \mathscr{I})$ is a semi$\mathscr{I}-T_{0}$ space which is not semi- $\mathscr{I}-T_{1}$.

Theorem 16. An ideal topological space $(X, \tau, \mathscr{I})$ is semi- $\mathscr{I}-T_{0}$ if and only if for each pair of distinct points $x, y$ in $X, s C l_{\mathscr{I}}(\{x\}) \neq s \mathrm{Cl}_{\mathscr{I}}(\{y\})$.

Proof. Suppose that $x, y \in X, x \neq y$ and $s \mathrm{Cl}_{\mathscr{I}}(\{x\}) \neq s \mathrm{Cl}_{\mathscr{I}}(\{y\})$. Let $z$ be a point of $X$ such that $z \in s \mathrm{Cl}_{\mathscr{I}}(\{x\})$ but $z \notin s \mathrm{Cl}_{\mathscr{I}}(\{y\})$. We claim that $x \notin s \mathrm{Cl}_{\mathscr{I}}(\{y\})$. For, if $x \in s \mathrm{Cl}_{\mathscr{I}}(\{y\})$, then $s \mathrm{Cl}_{\mathscr{I}}(\{x\}) \subseteq s \mathrm{Cl}_{\mathscr{I}}(\{y\})$. And this contradicts the fact that $z \notin s \mathrm{Cl}_{\mathscr{I}}(\{y\})$. Thus, $x$ belongs to the semi- $\mathscr{I}$-open set $X-s \mathrm{Cl}_{\mathscr{I}}(\{y\})$ to which $y$ does not belong.

Conversely, let $(X, \tau, \mathscr{I})$ be a semi- $\mathscr{I}-T_{0}$ space and $x, y$ be any two distinct points of $X$. There exists a semi- $\mathscr{I}$-open set $G$ containing $x$ or $y$, say $x$ but not $y$. Then $X-G$ is a semi- $\mathscr{I}$-closed set which does not contain $x$ but contains $y$. Since $s \mathrm{Cl}_{\mathscr{I}}(\{y\}) \subseteq X-G$ and hence $x \notin s \mathrm{Cl}_{\mathscr{I}}(\{y\})$. Consequently, we obtain $s \mathrm{Cl}_{\mathscr{I}}(\{x\}) \neq s \mathrm{Cl}_{\mathscr{I}}(\{y\})$.

Theorem 17. An ideal topological space $(X, \tau, \mathscr{I})$ is semi- $\mathscr{I}-T_{0}$ if and only if for each $x \in X$, the singleton $\{x\}$ is $\mathscr{S}^{\star}$-closed.

Proof. Suppose that $(X, \tau, \mathscr{I})$ is semi- $\mathscr{I}-T_{0}$. For each $x \in X$, it is obvious that

$$
\{x\} \subseteq \Lambda_{I}^{s}(\{x\}) \cap s \mathrm{Cl}_{\mathscr{I}}(\{x\}) .
$$

If $y \neq x,(i)$ there exists a semi- $\mathscr{I}$-open set $V_{x}$ such that $y \notin V_{x}$ and $x \in V_{x}$ of $(i i)$ there exists a semi- $\mathscr{I}$ open set $V_{y}$ such that $x \notin V_{y}$ and $y \in V_{y}$. In case of $(i), y \notin \Lambda_{I}^{s}(\{x\})$ and $y \notin \Lambda_{I}^{s}(\{x\}) \cap s \mathrm{Cl}_{\mathscr{I}}(\{x\})$. 
Therefore, $\{x\} \supseteq \Lambda_{I}^{s}(\{x\}) \cap s \mathrm{Cl}_{\mathscr{I}}(\{x\})$. In case (ii), $y \notin s \mathrm{Cl}_{\mathscr{I}}(\{x\})$ and $y \notin \Lambda_{I}^{s}(\{x\}) \cap s \mathrm{Cl}_{\mathscr{I}}(\{x\})$. This shows that $\{x\} \supseteq \Lambda_{I}^{s}(\{x\}) \cap s \mathrm{Cl}_{\mathscr{I}}(\{x\})$. Consequently, we obtain $\{x\}=\Lambda_{I}^{s}(\{x\}) \cap s \mathrm{Cl}_{\mathscr{I}}(\{x\})$.

Conversely, suppose that $(X, \tau, \mathscr{I})$ is not semi$\mathscr{I}-T_{0}$. There exist distinct points $x, y$ of $X$ such that (i) $y \in V_{x}$ for every semi- $\mathscr{I}$-open set $V_{x}$ containing $x$ and $(i i) x \in V_{y}$ for every semi- $\mathscr{I}$-open set $V_{y}$ containing $y$. From $(i)$ and $(i i)$, we obtain $y \in \Lambda_{I}^{s}(\{x\})$ and $y \in s \mathrm{Cl}_{\mathscr{I}}(\{x\})$, respectively. Therefore, we have $y \in \Lambda_{I}^{s}(\{x\}) \cap s \mathrm{Cl}_{\mathscr{I}}(\{x\})$. By Theorem 13, $\{x\}=\Lambda_{I}^{s}(\{x\}) \cap s \mathrm{Cl}_{\mathscr{I}}(\{x\})$, since $\{x\}$ is $\mathscr{S}^{\star}{ }_{-}$ closed. This is contrary $x \neq y$.

Definition 18. Let $A$ be a subset of an ideal topological space $(X, \tau, \mathscr{I})$. A subset $\delta_{\star}^{s}(A)$ is defined as follows: $\delta_{\star}^{s}(A)=\cup\{F \mid F \in s \mathscr{I} C(X, \tau), F \subseteq A\}$.

Lemma 19. For subsets $A, B$ and $\left\{C_{\gamma} \mid \gamma \in \Gamma\right\}$ of an ideal topological space $(X, \tau, \mathscr{I})$, the following properties hold:

(1) $\delta_{\star}^{s}(A) \subseteq A$.

(2) $\delta_{\star}^{s}\left(\delta_{\star}^{s}(A)\right)=\delta_{\star}^{s}(A)$.

(3) If $A \subseteq B$, then $\delta_{\star}^{s}(A) \subseteq \delta_{\star}^{s}(B)$.

(4) If $A \in s \mathscr{I} C(X, \tau)$, then $A=\delta_{\star}^{s}(A)$.

(5) $\underset{\gamma \in \Gamma}{\cup} \delta_{\star}^{s}\left(C_{\gamma}\right) \subseteq \delta_{\star}^{s}\left(\underset{\gamma \in \Gamma}{\cup} C_{\gamma}\right)$.

(6) $\delta_{\star}^{s}\left(\underset{\gamma \in \Gamma}{\cap} C_{\gamma}\right)=\bigcap_{\gamma \in \Gamma} \delta_{\star}^{s}\left(C_{\gamma}\right)$.

(7) $\Lambda_{I}^{s}(X-A)=X-\delta_{\star}^{s}(A)$ and

$$
\delta_{\star}^{s}(X-A)=X-\Lambda_{I}^{s}(A) .
$$

Definition 20. A subset $A$ of an ideal topological space $(X, \tau, \mathscr{I})$ is called $\delta_{\star}^{s}$-set if $A=\delta_{\star}^{s}(A)$. The family of all $\delta_{\star}^{s}$-sets of $(X, \tau, \mathscr{I})$ is denoted by $\delta_{\star}^{s}(X)$ (or simply $\delta_{\star}^{s}$ ).

Lemma 21. For subsets $A$ and $B_{\gamma}(\gamma \in \Gamma)$ of an ideal topological space $(X, \tau, \mathscr{I})$, the following properties hold:

(1) $\delta_{\star}^{s}(A)$ is a $\delta_{\star}^{s}$-set.

(2) If $A$ is a semi-I- -closed set, then $A$ is $\delta_{\star}^{s}$-set.

(3) If $B_{\gamma}$ is a $\delta_{\star}^{s}$-set for each $\gamma \in \Gamma$, then $\cup_{\gamma \in \Gamma} B_{\gamma}$ is $\delta_{\star}^{s}$-set.

(4) If $B_{\gamma}$ is a $\delta_{\star}^{s}$-set for each $\gamma \in \Gamma$, then $\cap_{\gamma \in \Gamma} B_{\gamma}$ is $\delta_{\star}^{s}$-set.
Definition 22. A subset $A$ of an ideal topological space $(X, \tau, \mathscr{I})$ is said to be $\mathscr{S}^{\star}$-open if the complement of $A$ is $\mathscr{S}^{\star}$-closed. The family of all $\mathscr{S}^{\star}$ open sets in an ideal topological space $(X, \tau, \mathscr{I})$ is denoted by $\mathscr{S}^{\star} O(X)$.

Theorem 23. For a subset $A$ of an ideal topological space $(X, \tau, \mathscr{I})$, the following properties are equivalent:

(1) A is $\mathscr{S}^{\star}$-open.

(2) $A=T \cup G$, where $T$ is a $\delta_{\star}^{s}$-set and $G$ is a semi$\mathscr{I}$-open set.

(3) $A=T \cup \operatorname{sint}_{\mathscr{I}}(A)$, where $T$ is a $\delta_{\star}^{s}$-set.

(4) $A=\delta_{\star}^{s}(A) \cup \operatorname{sint}_{\mathscr{I}}(A)$.

(5) $A \subseteq C l^{\star}(\operatorname{Int}(A)) \cup \delta_{\star}^{s}(A)$.

Proof. (1) $\Rightarrow(2)$ : Suppose that $A$ is $\mathscr{S}^{\star}$-open. Then $X-A$ is $\mathscr{S}^{\star}$-closed and hence $X-A=T \cap F$, where $T$ is a $\Lambda_{I}^{s}$-set and $F$ is a semi- $\mathscr{I}$-closed set. Thus, we have $A=(X-T) \cup(X-F)$, where $X-T$ is a $\delta_{\star}^{s}$-set and $X-F$ is a semi- $\mathscr{I}$-open set.

$(2) \Rightarrow(3)$ : Let $A=T \cup G$, where $T$ is a $\delta_{\star}^{s}$-set and $G$ is a semi- $\mathscr{I}$-open set. Since $G \subseteq A$ and $G$ is semi- $\mathscr{I}$-open, $G \subseteq s \operatorname{Int} \mathscr{I}(A)$ and hence

$$
\begin{aligned}
A & =T \cup G \\
& \subseteq T \cup s \operatorname{Int}_{\mathscr{I}}(A) \\
& \subseteq A .
\end{aligned}
$$

Consequently, we obtain $A=T \cup s \operatorname{Int} \mathscr{I}(A)$.

$(3) \Rightarrow(4)$ : Let $A=T \cup s \operatorname{Int}_{\mathscr{I}}(A)$, where $T$ is a $\delta_{\star}^{s}$-set. Since $T \subseteq A$, we have $\delta_{\star}^{s}(A) \supseteq \delta_{\star}^{s}(T)$ and hence

$$
\begin{aligned}
A & \supseteq \delta_{\star}^{s}(A) \cup s \operatorname{Int}_{\mathscr{I}}(A) \\
& \supseteq \delta_{\star}^{s}(T) \cup s \operatorname{Int}_{\mathscr{I}}(A) \\
& =T \cup s \operatorname{Int}_{\mathscr{I}}(A) \\
& =A .
\end{aligned}
$$

This shows that $A=\delta_{\star}^{s}(A) \cup s \operatorname{Int}_{\mathscr{I}}(A)$.

$(4) \Rightarrow(5)$ : Let $A=\delta_{\star}^{s}(A) \cup s \operatorname{Int}_{\mathscr{I}}(A)$. By $(4)$ and Lemma 12(2), we have

$$
\begin{aligned}
A & =\delta_{\star}^{s}(A) \cup s \operatorname{Int} \mathscr{I}(A) \\
& =\delta_{\star}^{s}(A) \cup\left[A \cap \mathrm{Cl}^{\star}(\operatorname{Int}(A))\right] \\
& =\left[\delta_{\star}^{s}(A) \cup A\right] \cap\left[\mathrm{Cl}^{\star}(\operatorname{Int}(A)) \cup \delta_{\star}^{s}(A)\right] \\
& =A \cap\left[\mathrm{Cl}^{\star}(\operatorname{Int}(A)) \cup \delta_{\star}^{s}(A)\right]
\end{aligned}
$$

and hence $A \subseteq \mathrm{Cl}^{\star}(\operatorname{Int}(A)) \cup \delta_{\star}^{s}(A)$. 
$(5) \Rightarrow(1)$ : Let $A \subseteq \mathrm{Cl}^{\star}(\operatorname{Int}(A)) \cup \delta_{\star}^{s}(A)$. Then

$$
\begin{aligned}
& \operatorname{Int}^{\star}(\mathrm{Cl}(X-A)) \cap \Lambda_{I}^{s}(X-A) \\
& =\left(X-\mathrm{Cl}^{\star}(\operatorname{Int}(A))\right) \cap\left(X-\delta_{\star}^{s}(A)\right) \\
& =X-\left(\mathrm{Cl}^{\star}(\operatorname{Int}(A)) \cup \delta_{\star}^{s}(A)\right) \\
& \subseteq X-A
\end{aligned}
$$

and by Theorem 13, we have $X-A$ is $\mathscr{S}^{\star}$-closed. Thus, $A$ is $\mathscr{S}^{\star}$-open.

Proposition 24. For subset $A_{\gamma}(\gamma \in \Gamma)$ of an ideal topological space $(X, \tau, \mathscr{I})$, the following properties hold:

(1) If $A_{\gamma}$ is $\mathscr{S}^{\star}$-closed for each $\gamma \in \Gamma$, then $\cap_{\gamma \in \Gamma} A_{\gamma}$ is $\mathscr{S}^{\star}$-closed.

(2) If $A_{\gamma}$ is $\mathscr{S}^{\star}$-open for each $\gamma \in \Gamma$, then $\cup_{\gamma \in \Gamma} A_{\gamma}$ is $\mathscr{S}^{\star}$-open.

\section{Characterizations of semi- $\mathscr{I}-R_{0}$ ideal topological spaces}

In this section, we introduce the notion of semi- $\mathscr{I}$ $R_{0}$ ideal topological spaces. Moreover, some characterizations of semi- $\mathscr{I}-R_{0}$ ideal topological spaces are discussed.

Definition 25. An ideal topological space $(X, \tau, \mathscr{I})$ is said to be semi- $\mathscr{I}-R_{0}$ if for each semi- $\mathscr{I}$-open set $U$ and each $x \in U, s l_{\mathscr{I}}(\{x\}) \subseteq U$.

Proposition 26. An ideal topological space $(X, \tau, \mathscr{I})$ is semi- $\mathscr{I}-R_{0}$ if and only if for each $U \in s \mathscr{I} O(X, \tau)$, $x \in U$ implies $\operatorname{Int}^{\star}(C l(\{x\})) \subseteq U$.

Proof. This is obvious by Lemma 12(1).

Theorem 27. For an ideal topological space $(X, \tau, \mathscr{I})$, the following properties are equivalent:

(1) $(X, \tau, \mathscr{I})$ is semi- $\mathscr{I}-R_{0}$.

(2) For each $x, y \in X, s C l_{\mathscr{I}}(\{x\}) \neq s \mathrm{Cl}_{\mathscr{I}}(\{y\})$ implies $\mathrm{sCl}_{\mathscr{I}}(\{x\}) \cap \mathrm{sCl}_{\mathscr{I}}(\{y\})=\emptyset$.

Proof. (1) $\Rightarrow(2)$ : Suppose that $(X, \tau, \mathscr{I})$ is semi$\mathscr{I}-R_{0}$ and $x, y \in X$ such that

$$
s \mathrm{Cl}_{\mathscr{I}}(\{x\}) \neq s \mathrm{Cl}_{\mathscr{I}}(\{y\}) .
$$

Then, there exists $z \in s \mathrm{Cl}_{\mathscr{I}}(\{x\})$ such that

$$
z \notin s \mathrm{Cl}_{\mathscr{I}}(\{y\})
$$

(or $z \in s \mathrm{Cl}_{\mathscr{I}}(\{y\})$ such that $z \notin s \mathrm{Cl}_{\mathscr{I}}(\{x\})$ ). There exists $V \in s \mathscr{I} O(X, \tau)$ such that $y \notin V$ and $z \in V$; hence $x \in V$. Therefore, we have $x \notin s \mathrm{Cl}_{\mathscr{I}}(\{y\})$. Thus, $x \in X-s \mathrm{Cl}_{\mathscr{I}}(\{y\}) \in s \mathscr{I} O(X, \tau)$, which implies $s \mathrm{Cl}_{\mathscr{I}}(\{x\}) \subseteq X-s \mathrm{Cl}_{\mathscr{I}}(\{y\})$ and hence $s \mathrm{Cl}_{\mathscr{I}}(\{x\}) \cap s \mathrm{Cl}_{\mathscr{I}}(\{y\})=\emptyset$. The proof for otherwise is similar.

$(2) \Rightarrow(1)$ : Let $V \in s \mathscr{I} O(X, \tau)$ and $x \in V$. If $y \notin V$, then $s \mathrm{Cl}_{\mathscr{I}}(\{y\}) \cap V=\emptyset$. Therefore,

$$
x \notin s \mathrm{Cl}_{\mathscr{I}}(\{y\})
$$

and $s \mathrm{Cl}_{\mathscr{I}}(\{x\}) \neq s \mathrm{Cl}_{\mathscr{I}}(\{y\})$. By the hypothesis, $s \mathrm{Cl}_{\mathscr{I}}(\{x\}) \cap s \mathrm{Cl}_{\mathscr{I}}(\{y\})=\emptyset$ and hence

$$
y \notin s \mathrm{Cl}_{\mathscr{I}}(\{x\}) .
$$

This shows that $s \mathrm{Cl}_{\mathscr{I}}(\{x\}) \subseteq V$. Thus, $(X, \tau, \mathscr{I})$ is semi- $\mathscr{I}-R_{0}$.

Lemma 28. For each points $x, y$ of an ideal topological space $(X, \tau, \mathscr{I})$, the following properties hold:

(1) $y \in \Lambda_{I}^{s}(\{x\})$ if and only if $x \in s C l_{\mathscr{I}}(\{y\})$.

(2) $\Lambda_{I}^{s}(\{x\})=\Lambda_{I}^{s}(\{y\})$ if and only if

$$
s C l_{\mathscr{I}}(\{x\})=s C l_{\mathscr{I}}(\{y\}) .
$$

Proof. (1) Let $x \notin s \mathrm{Cl}_{\mathscr{I}}(\{y\})$. Then, there exists $U \in s \mathscr{I} O(X, \tau)$ such that $x \in U$ and $y \notin U$. Thus, $y \notin \Lambda_{I}^{s}(\{x\})$. The converse is similarly shown.

(2) Suppose that $\Lambda_{I}^{s}(\{x\})=\Lambda_{I}^{s}(\{x\})$ for any points $x, y$ in $X$. Since $x \in \Lambda_{I}^{s}(\{x\})$, we have $x \in \Lambda_{I}^{s}(\{y\})$ and by (1), $y \in s \mathrm{Cl}_{\mathscr{I}}(\{x\})$. Therefore, $s \mathrm{Cl}_{\mathscr{I}}(\{y\}) \subseteq s \mathrm{Cl}_{\mathscr{I}}(\{x\})$. Similarly, we have $s \mathrm{Cl}_{\mathscr{I}}(\{x\}) \subseteq s \mathrm{Cl}_{\mathscr{I}}(\{y\})$ and hence $s \mathrm{Cl}_{\mathscr{I}}(\{x\})=$ $s \mathrm{Cl}_{\mathscr{I}}(\{y\})$.

Conversely, suppose that

$$
s \mathrm{Cl}_{\mathscr{I}}(\{x\})=s \mathrm{Cl}_{\mathscr{I}}(\{y\}) .
$$

Since $x \in s \mathrm{Cl}_{\mathscr{I}}(\{x\}), x \in s \mathrm{Cl}_{\mathscr{I}}(\{y\})$ and by (1), $y \in \Lambda_{I}^{s}(\{x\})$. By Lemma 4,

$$
\begin{aligned}
\Lambda_{I}^{s}(\{y\}) & \subseteq \Lambda_{I}^{s}\left(\Lambda_{I}^{s}(\{x\})\right) \\
& =\Lambda_{I}^{s}(\{x\}) .
\end{aligned}
$$

Similarly, we have $\Lambda_{I}^{s}(\{x\}) \subseteq \Lambda_{I}^{s}(\{y\})$ and hence

$$
\Lambda_{I}^{s}(\{x\})=\Lambda_{I}^{s}(\{y\}) .
$$

Theorem 29. For an ideal topological space $(X, \tau, \mathscr{I})$, the following properties are equivalent:

(1) $(X, \tau, \mathscr{I})$ is semi- $\mathscr{I}-R_{0}$. 
(2) For each $x, y \in X, \Lambda_{I}^{s}(\{x\}) \neq \Lambda_{I}^{s}(\{y\})$ implies $\Lambda_{I}^{s}(\{x\}) \cap \Lambda_{I}^{s}(\{y\})=\emptyset$.

Proof. (1) $\Rightarrow(2)$ : Let $(X, \tau, \mathscr{I})$ be semi- $\mathscr{I}-R_{0}$ and $\Lambda_{I}^{s}(\{x\}) \neq \Lambda_{I}^{s}(\{y\})$ for any points $x, y$ in $X$. Suppose that $\Lambda_{I}^{s}(\{x\}) \cap \Lambda_{I}^{s}(\{y\}) \neq \emptyset$. Let $z \in \Lambda_{I}^{s}(\{x\}) \cap \Lambda_{I}^{s}(\{y\})$. Then $z \in \Lambda_{I}^{s}(\{x\})$ and by Lemma 28(1), $x \in s \mathrm{Cl}_{\mathscr{I}}(\{z\})$. Therefore, $x \in s \mathrm{Cl}_{\mathscr{I}}(\{z\}) \cap s \mathrm{Cl}_{\mathscr{I}}(\{x\})$ and by Theorem 27, $s \mathrm{Cl}_{\mathscr{I}}(\{z\})=s \mathrm{Cl}_{\mathscr{I}}(\{x\})$. Similarly, we have $s \mathrm{Cl}_{\mathscr{I}}(\{z\})=s \mathrm{Cl}_{\mathscr{I}}(\{y\})$ and hence

$$
s \mathrm{Cl}_{\mathscr{I}}(\{x\})=s \mathrm{Cl}_{\mathscr{I}}(\{y\}) .
$$

By Lemma 28(2), we obtain $\Lambda_{I}^{s}(\{x\})=\Lambda_{I}^{s}(\{y\})$.

$(2) \Rightarrow(1)$ : We show the sufficiency by using Theorem 27. Suppose that $s \mathrm{Cl}_{\mathscr{I}}(\{x\}) \neq s \mathrm{Cl}_{\mathscr{I}}(\{y\})$. By Lemma 28(2), we have $\Lambda_{I}^{s}(\{x\}) \neq \Lambda_{I}^{s}(\{y\})$ and hence $\Lambda_{I}^{s}(\{x\}) \cap \Lambda_{I}^{s}(\{y\})=\emptyset$. Thus,

$$
s \mathrm{Cl}_{\mathscr{I}}(\{x\}) \cap s \mathrm{Cl}_{\mathscr{I}}(\{y\})=\emptyset .
$$

In fact, assume $z \in s \mathrm{Cl}_{\mathscr{I}}(\{x\}) \cap s \mathrm{Cl}_{\mathscr{I}}(\{y\})$. Then $z \in s \mathrm{Cl}_{\mathscr{I}}(\{x\})$ implies $x \in \Lambda_{I}^{s}(\{z\})$ and hence

$$
x \in \Lambda_{I}^{s}(\{z\}) \cap \Lambda_{I}^{s}(\{x\}) .
$$

By the hypothesis, $\Lambda_{I}^{s}(\{z\})=\Lambda_{I}^{s}(\{x\})$ and by Lemma 28(2), $s \mathrm{Cl}_{\mathscr{I}}(\{z\})=s \mathrm{Cl}_{\mathscr{I}}(\{x\})$. Similarly, we have $s \mathrm{Cl}_{\mathscr{I}}(\{z\})=s \mathrm{Cl}_{\mathscr{I}}(\{y\})$ and hence $s \mathrm{Cl}_{\mathscr{I}}(\{x\})=s \mathrm{Cl}_{\mathscr{I}}(\{y\})$. This contradicts that $s \mathrm{Cl}_{\mathscr{I}}(\{x\}) \neq s \mathrm{Cl}_{\mathscr{I}}(\{y\})$. Thus,

$$
s \mathrm{Cl}_{\mathscr{I}}(\{x\}) \cap s \mathrm{Cl}_{\mathscr{I}}(\{y\})=\emptyset .
$$

This shows that $(X, \tau, \mathscr{I})$ is semi- $\mathscr{I}-R_{0}$.

Theorem 30. For an ideal topological space $(X, \tau, \mathscr{I})$, the following properties are equivalent:

(1) $(X, \tau, \mathscr{I})$ is semi- $\mathscr{I}-R_{0}$.

(2) $x \in s \mathrm{sl}_{\mathscr{I}}(\{y\})$ if and only if $y \in s C l_{\mathscr{I}}(\{x\})$.

Proof. (1) $\Rightarrow(2)$ : Suppose that $x \in s \mathrm{Cl}_{\mathscr{I}}(\{y\})$. By Lemma 28(1), $y \in \Lambda_{I}^{s}(\{x\})$ and hence

$$
\Lambda_{I}^{s}(\{x\}) \cap \Lambda_{I}^{s}(\{y\}) \neq \emptyset .
$$

By Theorem 29, we have $\Lambda_{I}^{s}(\{x\})=\Lambda_{I}^{s}(\{y\})$ and hence $x \in \Lambda_{I}^{s}(\{y\})$. By Lemma 28(1),

$$
y \in s \mathrm{Cl}_{\mathscr{I}}(\{x\}) .
$$

The converse is similarly shown.

$(2) \Rightarrow(1)$ : Let $U \in s \mathscr{I} O(X, \tau)$ and $x \in U$. If $y \notin U$, then $s \mathrm{Cl}_{\mathscr{I}}(\{y\}) \cap U=\emptyset$. Hence, $x \notin s \mathrm{Cl}_{\mathscr{I}}(\{y\})$ and $y \notin s \mathrm{Cl}_{\mathscr{I}}(\{x\})$. This implies that $s \mathrm{Cl}_{\mathscr{I}}(\{x\}) \subseteq U$. Thus, $(X, \tau, \mathscr{I})$ is semi- $\mathscr{I}$ $R_{0}$.
Theorem 31. For an ideal topological space $(X, \tau, \mathscr{I})$, the following properties are equivalent:

(1) $(X, \tau, \mathscr{I})$ is semi- $\mathscr{I}-T_{1}$.

(2) For each point $x \in X,\{x\}$ is semi- $\mathscr{I}$-closed.

(3) $(X, \tau, \mathscr{I})$ is semi- $\mathscr{I}-R_{0}$ and semi- $\mathscr{I}-T_{0}$.

Proof. $(1) \Rightarrow(2)$ : It follows from Lemma 9.

$(2) \Rightarrow(3)$ : The proof is obvious.

$(3) \Rightarrow(1)$ : Let $x$ and $y$ be any distinct points of $X$. Since $(X, \tau, \mathscr{I})$ is a semi- $\mathscr{I}-T_{0}$ space, there exists a semi- $\mathscr{I}$-open set $U$ such that either $x \in U$ and $y \notin U$ or $x \notin U$ and $y \in U$. In case $x \in U$ and $y \notin U$, we have $x \in s \mathrm{Cl}_{\mathscr{I}}(\{x\}) \subseteq U$ and hence $y \in X-U \subseteq X-s \mathrm{Cl}_{\mathscr{I}}(\{x\})$. Since the proof of the other is quite similar, $(X, \tau, \mathscr{I})$ is a semi- $\mathscr{I}-T_{1}$ space.

Theorem 32. For an ideal topological space $(X, \tau, \mathscr{I})$, the following properties are equivalent:

(1) $(X, \tau, \mathscr{I})$ is semi- $\mathscr{I}-R_{0}$.

(2) For any nonempty set $A$ and each

$$
G \in s \mathscr{I} O(X, \tau)
$$

such that $A \cap G \neq \emptyset$, there exists a semi- $\mathscr{I}$ closed set $F$ such that $A \cap F \neq \emptyset$ and $F \subseteq G$.

(3) For each semi-I -open set $G$,

$$
G=\cup\{F \mid F \in s \mathscr{I} C(X, \tau), F \subseteq G\} .
$$

(4) For each semi- $\mathscr{I}$-closed set $F, F=\Lambda_{I}^{s}(F)$.

(5) For each $x \in X, s l_{\mathscr{I}}(\{x\}) \subseteq \Lambda_{I}^{s}(\{x\})$.

(6) For each semi- $\mathscr{I}$-closed set $F$ and each

$$
x \in X-F,
$$

$F \cap s C l_{\mathscr{I}}(\{x\})=\emptyset$.

(7) For any distinct points $x$ and $y$ of $X$, $s l_{\mathscr{I}}(\{x\})=s l_{\mathscr{I}}(\{y\})$ or

$$
s C l_{\mathscr{I}}(\{x\}) \cap s C l_{\mathscr{I}}(\{y\})=\emptyset .
$$

(8) For each semi- $\mathscr{I}$-closed set $F$ and each

$$
x \in X-F,
$$

there exists a semi- $\mathscr{I}$-open set $U$ such that $F \subseteq$ $U$ and $x \notin U$.

(9) For each semi- $\mathscr{I}$-closed set $F$ and each $x \in F$, $\Lambda_{I}^{s}(\{x\}) \subseteq F$. 
(10) For each $x \in X, \Lambda_{I}^{s}(\{x\}) \subseteq s l_{\mathscr{I}}(\{x\})$.

Proof. (1) $\Rightarrow(2)$ : Let $A$ be a nonempty set of $X$ and $G \in s \mathscr{I} O(X, \tau)$ such that $A \cap G \neq \emptyset$. Then, there exists $x \in A \cap G$. Since $x \in G \in s \mathscr{I} O(X, \tau)$, we have $s \mathrm{Cl}_{\mathscr{I}}(\{x\}) \subseteq G$. Put $F=s \mathrm{Cl}_{\mathscr{I}}(\{x\})$, then $F \in s \mathscr{I} C(X, \tau), A \cap F \neq \emptyset$ and $F \subseteq G$.

$(2) \Rightarrow(3):$ Let $G \in s \mathscr{I} O(X, \tau)$, then

$$
G \supseteq \cup\{F \mid F \in \operatorname{sIC}(X, \tau), F \subseteq G\} .
$$

Let $x \in G$. Then, there exists $F \in s \mathscr{I} C(X, \tau)$ such that $x \in F$ and $F \subseteq G$. Therefore,

$$
x \in \cup\{F \mid F \in s \mathscr{I} C(X, \tau), F \subseteq G\}
$$

and hence $G \subseteq \cup\{F \mid F \in s \mathscr{I} C(X, \tau), F \subseteq G\}$.

$(3) \Rightarrow(4)$ : Let $F \in s \mathscr{I} C(X, \tau)$. By Lemma 4, we have $F \subseteq \Lambda_{I}^{s}(F)$. Next, we show $F \supseteq \Lambda_{I}^{s}(F)$. Let $x \notin F$. Then, we have $x \in X-F \in s \mathscr{I} O(X, \tau)$ and by (3), there exists a semi- $\mathscr{I}$-closed set $K$ such that $x \in K$ and $K \subseteq X-F$. Now, put $U=X-K$. Then $F \subseteq U \in s \mathscr{I} O(X, \tau)$ and $x \notin U$. Thus, $x \notin \Lambda_{I}^{s}(F)$. This shows that $F \supseteq \Lambda_{I}^{s}(F)$.

$(4) \Rightarrow(5)$ : Let $x \in X$ and $y \notin \Lambda_{I}^{s}(\{x\})$. There exists $U \in s \mathscr{I} O(X, \tau)$ such that $x \in U$ and $y \notin U$. Thus, $s \mathrm{Cl}_{\mathscr{I}}(\{y\}) \cap U=\emptyset$. By (4), we have

$$
\Lambda_{I}^{s}\left(s \mathrm{Cl}_{\mathscr{I}}(\{y\})\right) \cap U=\emptyset .
$$

Since $x \notin \Lambda_{I}^{s}\left(s \mathrm{Cl}_{\mathscr{I}}(\{y\})\right)$, there exists

$$
V \in s \mathscr{I} O(X, \tau)
$$

such that $s \mathrm{Cl}_{\mathscr{I}}(\{y\}) \subseteq V$ and $x \notin V$. Therefore, $s \mathrm{Cl}_{\mathscr{I}}(\{x\}) \cap V=\emptyset$. Since $y \in V, y \notin s \mathrm{Cl}_{\mathscr{I}}(\{x\})$ and hence $s \mathrm{Cl}_{\mathscr{I}}(\{x\}) \subseteq \Lambda_{I}^{s}(\{x\})$.

$(5) \Rightarrow(6)$ : Let $F$ be a semi- $\mathscr{I}$-closed set such that $x \in X-F$. By (5), we have

$$
\begin{aligned}
s \mathrm{Cl}_{\mathscr{I}}(\{x\}) & \subseteq \Lambda_{I}^{s}(\{x\}) \\
& \subseteq \Lambda_{I}^{s}(X-F) \\
& =X-F
\end{aligned}
$$

and hence $s \mathrm{Cl}_{\mathscr{I}}(\{x\}) \cap F=\emptyset$.

$(6) \Rightarrow(7)$ : Let $x, y$ be distinct points of $X$. Suppose that $s \mathrm{Cl}_{\mathscr{I}}(\{x\}) \cap s \mathrm{Cl}_{\mathscr{I}}(\{y\}) \neq \emptyset$. By (6), we have $x \in s \mathrm{Cl}_{\mathscr{I}}(\{y\})$ and $y \in s \mathrm{Cl}_{\mathscr{I}}(\{x\})$. Thus, $s \mathrm{Cl}_{\mathscr{I}}(\{x\}) \subseteq s \mathrm{Cl}_{\mathscr{I}}(\{y\}) \subseteq s \mathrm{Cl}_{\mathscr{I}}(\{x\})$. Consequently, we obtain $s \mathrm{Cl}_{\mathscr{I}}(\{x\})=s \mathrm{Cl}_{\mathscr{I}}(\{y\})$.

$(7) \Rightarrow(1)$ : Let $V \in s \mathscr{I} O(X, \tau)$ and $x \in V$. For each $y \notin V, s \mathrm{Cl}_{\mathscr{I}}(\{y\}) \cap V=\emptyset$ and hence

$$
x \notin s \mathrm{Cl}_{\mathscr{I}}(\{y\}) .
$$

Therefore, $s \mathrm{Cl}_{\mathscr{I}}(\{x\}) \neq s \mathrm{Cl}_{\mathscr{I}}(\{y\})$. By (7), for each $y \notin V, s \mathrm{Cl}_{\mathscr{I}}(\{x\}) \cap s \mathrm{Cl}_{\mathscr{I}}(\{y\})=\emptyset$. Since
$X-V$ is semi- $\mathscr{I}$-closed, $y \in s \mathrm{Cl}_{\mathscr{I}}(\{y\}) \subseteq X-V$ and $\cup_{y \in X-U} s \mathrm{Cl}_{\mathscr{I}}(\{y\})=X-U$. Therefore,

$$
\begin{aligned}
& s \mathrm{Cl}_{\mathscr{I}}(\{x\}) \cap(X-U) \\
& =s \mathrm{Cl}_{\mathscr{I}}(\{x\}) \cap\left[\cup_{y \in X-U} s \mathrm{Cl}_{\mathscr{I}}(\{y\})\right] \\
& =\cup_{y \in X-U}\left[s \mathrm{Cl}_{\mathscr{I}}(\{x\}) \cap s \mathrm{Cl}_{\mathscr{I}}(\{y\})\right] \\
& =\emptyset .
\end{aligned}
$$

Thus, $s \mathrm{Cl}_{\mathscr{I}}(\{x\}) \subseteq V$. This shows that $(X, \tau, \mathscr{I})$ is semi- $\mathscr{I}-R_{0}$.

$(1) \Rightarrow(8)$ : Let $F$ be a semi- $\mathscr{I}$-closed set such that $x \in X-F$. Since $(X, \tau, \mathscr{I})$ is semi- $\mathscr{I}-R_{0}$, we have $s \mathrm{Cl}_{\mathscr{I}}(\{x\}) \subseteq X-F$. Put $U=X-s \mathrm{Cl}_{\mathscr{I}}(\{x\})$, then $U \in s \mathscr{I} O(X, \tau), F \subseteq U$ and $x \notin U$.

$(8) \Rightarrow(9)$ : Let $F$ be a semi- $\mathscr{I}$-closed set such that $x \in F$. Let $y \notin F$. Then $y \in X-F$ and by (8), there exists a semi- $\mathscr{I}$-open set $U$ such that $F \subseteq U$ and $y \notin U$. Therefore, $y \notin \Lambda_{I}^{s}(F)$ and hence $y \notin \Lambda_{I}^{s}(\{x\})$. This shows that $\Lambda_{I}^{s}(\{x\}) \subseteq F$.

$(9) \Rightarrow(10)$ : Let $x$ be any point of $X$. Since $x \in s \mathrm{Cl}_{\mathscr{I}}(\{x\})$ and $s \mathrm{Cl}_{\mathscr{I}}(\{x\})$ is semi- $\mathscr{I}$-closed, by $(9), \Lambda_{I}^{s}(\{x\}) \subseteq s \mathrm{Cl}_{\mathscr{I}}(\{x\})$.

$(10) \Rightarrow(1)$ : Let $U \in s \mathscr{I} O(X, \tau)$ and $x \in U$. If $y \notin U$, then $s \mathrm{Cl}_{\mathscr{I}}(\{y\}) \cap U=\emptyset$ and $x \notin s \mathrm{Cl}_{\mathscr{I}}(\{y\})$. By (10), we have $x \notin \Lambda_{I}^{s}(\{y\})$ and by Lemma 28(1), $y \notin s \mathrm{Cl}_{\mathscr{I}}(\{x\})$. Thus, $s \mathrm{Cl}_{\mathscr{I}}(\{x\}) \subseteq U$. This shows that $(X, \tau, \mathscr{I})$ is semi- $\mathscr{I}-R_{0}$.

For a subset $A$ of an ideal topological space $(X, \tau, \mathscr{I})$, we denote by $\tau_{\left.\right|_{A}}$ the relative topology on $A$ and $\mathscr{I}_{\left.\right|_{A}}=\left\{A \cap I_{0} \mid I_{0} \in \mathscr{I}\right\}$ is an ideal on $A$.

Lemma 33. [6] Let $(X, \tau, \mathscr{I})$ be an ideal topological space and $B \subseteq A \subseteq X$. Then

$$
B^{\star}\left(\tau_{\left.\right|_{A}}, \mathscr{I}_{\left.\right|_{A}}\right)=B^{\star}(\tau, \mathscr{I}) \cap A .
$$

Lemma 34. [11] Let $(X, \tau, \mathscr{I})$ be an ideal topological space and $B \subseteq A \subseteq X$. Then

$$
C l_{A}^{\star}(B)=C l^{\star}(B) \cap A .
$$

Lemma 35. [11] Let $A$ be a subset of an ideal topological space $(X, \tau, \mathscr{I})$ and $U$ be an open set. Then $U \cap C l^{\star}(A) \subseteq C l^{\star}(U \cap A)$.

Lemma 36. Let $(X, \tau, \mathscr{I})$ be an ideal topological space and $A \subseteq U \in \tau$. If $A$ is semi- $\mathscr{I}_{\left.\right|_{U}}$-open in $\left(U, \tau_{\left.\right|_{U}}, \mathscr{I}_{\left.\right|_{U}}\right)$, then $A$ is semi- $\mathscr{I}$-open in $(X, \tau, \mathscr{I})$.

Proof. Let $A$ be semi- $\mathscr{I}_{\left.\right|_{U}}$-open in $\left(U, \tau_{\left.\right|_{U}}, \mathscr{I}_{\left.\right|_{U}}\right)$. By 
Lemma 34 and 35, we have

$$
\begin{aligned}
A & \subseteq \mathrm{Cl}_{U}^{\star}\left(\operatorname{Int}_{U}(A)\right) \\
& =\mathrm{Cl}^{\star}\left(\operatorname{Int}_{U}(A)\right) \cap U \\
& =\mathrm{Cl}^{\star}(\operatorname{Int}(A)) \cap U \\
& \subseteq \mathrm{Cl}^{\star}(\operatorname{Int}(A) \cap U) \\
& =\mathrm{Cl}^{\star}(\operatorname{Int}(A \cap U)) \\
& \subseteq \mathrm{Cl}^{\star}(\operatorname{Int}(A)) .
\end{aligned}
$$

Therefore, $A$ is semi- $\mathscr{I}$-open in $(X, \tau, \mathscr{I})$.

Lemma 37. Let $(X, \tau, \mathscr{I})$ be an ideal topological space and $A \subseteq U \in \tau$. Then

$$
s C l_{\mathscr{I}_{U}}(A)=s C l_{\mathscr{I}}(A) \cap U .
$$

Proof. Let $A \subseteq U \in \tau$. By Lemma 12(1), we have

$$
\begin{aligned}
s \mathrm{Cl}_{\mathscr{I}_{U}}(A) & =A \cup \operatorname{Int}_{U}^{\star}\left(\mathrm{Cl}_{U}(A)\right) \\
& =A \cup \operatorname{Int}_{U}^{\star}(\mathrm{Cl}(A) \cap U) \\
& =A \cup \operatorname{Int}^{\star}(\mathrm{Cl}(A) \cap U) \\
& =A \cup\left(\operatorname{Int}^{\star}(\mathrm{Cl}(A)) \cap U\right) \\
& =\left(A \cup \operatorname{Int}^{\star}(\mathrm{Cl}(A))\right) \cap U \\
& =s \operatorname{Cl}_{\mathscr{I}}(A) \cap U .
\end{aligned}
$$

Theorem 38. Let $(X, \tau, \mathscr{I})$ be an ideal topological space and $U \in \tau$. If $(X, \tau, \mathscr{I})$ is semi- $\mathscr{I}-R_{0}$, then $\left(U, \tau_{\left.\right|_{U}}, \mathscr{I}_{\left.\right|_{U}}\right)$ is semi- $\mathscr{I}_{\left.\right|_{U}}-R_{0}$.

Proof. Suppose that $(X, \tau, \mathscr{I})$ is a semi- $\mathscr{I}-R_{0}$ space. Let $V \in s \mathscr{I}_{\left.\right|_{U}} O\left(U, \tau_{\left.\right|_{U}}\right)$ and $x \in V$. By Lemma 36 , we have $V \in s \mathscr{I} O(X, \tau)$. Since $(X, \tau, \mathscr{I})$ is semi- $\mathscr{I}-R_{0}, s \mathrm{Cl}_{\mathscr{I}}(\{x\}) \subseteq V$ and by Lemma 37 , $s \mathrm{Cl}_{\mathscr{I}_{U}}(\{x\})=s \mathrm{Cl}_{\mathscr{I}}(\{x\}) \cap U \subseteq V$. This shows that $\left(U, \tau_{\left.\right|_{U}}, \mathscr{I}_{\left.\right|_{U}}\right)$ is semi- $\mathscr{I}_{\left.\right|_{U}}-R_{0}$.

Definition 39. An ideal topological space $(X, \tau, \mathscr{I})$ is said to be weak semi- $\mathscr{I}-R_{0}$ if every $\mathscr{S}^{\star}$-closed singleton is a $\Lambda_{I}^{s}$-set.

Theorem 40. If $(X, \tau, \mathscr{I})$ is semi- $\mathscr{I}-R_{0}$, then $(X, \tau, \mathscr{I})$ is weak semi- $\mathscr{I}-R_{0}$.

Proof. Let $x \in X$ be such that $\{x\}$ is $\mathscr{S}^{\star}$-closed. By Theorem 13, we have $\{x\}=\Lambda_{I}^{s}(\{x\}) \cap s \mathrm{Cl}_{\mathscr{I}}(\{x\})$. Suppose that $\{x\}$ is not a $\Lambda_{I}^{s}$-set. Then, there exists $y \in \Lambda_{I}^{s}(\{x\})-\{x\}$. Thus, $y \notin s \mathrm{Cl}_{\mathscr{I}}(\{x\})$. Since $(X, \tau, \mathscr{I})$ is semi- $\mathscr{I}-R_{0}$ and by Theorem 27, $s \mathrm{Cl}_{\mathscr{I}}(\{y\}) \cap s \mathrm{Cl}_{\mathscr{I}}(\{x\})=\emptyset$. This shows that

$$
x \notin s \mathrm{Cl}_{\mathscr{I}}(\{y\}) .
$$

There exists a semi- $\mathscr{I}$-open set $V$ containing $x$ but not $y$ and hence $y \notin \Lambda_{I}^{s}(\{x\})$, which is a contradiction. Thus, $(X, \tau, \mathscr{I})$ is a weak semi- $\mathscr{I}-R_{0}$ space.

The converse of Theorem 40 need not be true as shown in the following example.

Example 41. Let $X=\{a, b, c\}$ with a topology $\tau=$ $\{\emptyset,\{a\}, X\}$ and an ideal $\mathscr{I}=\{\emptyset\}$. Then $(X, \tau, \mathscr{I})$ is a weak semi- $\mathscr{I}-R_{0}$ space which is not semi- $\mathscr{I}-R_{0}$.

Theorem 42. For an ideal topological space $(X, \tau, \mathscr{I})$, the following properties are equivalent:

(1) $(X, \tau, \mathscr{I})$ is semi- $\mathscr{I}-T_{1}$.

(2) $(X, \tau, \mathscr{I})$ is semi- $\mathscr{I}-T_{0}$ and semi- $\mathscr{I}-R_{0}$.

(3) $(X, \tau, \mathscr{I})$ is semi- $\mathscr{I}-T_{0}$ and weak semi- $\mathscr{I}-R_{0}$.

Proof. It suffices to show that $(3) \Rightarrow(1)$ : To shows that $(X, \tau, \mathscr{I})$ is semi- $\mathscr{I}-T_{1}$, it suffices to show by Lemma 9 that every singleton of $X$ is a $\Lambda_{I}^{s}$-set. Let $\{x\}$ be singleton of $X$. Since $(X, \tau, \mathscr{I})$ is semi- $\mathscr{I}$ $T_{0}$, it follows from Theorem 17 that $\{x\}$ is $\mathscr{S}^{\star}$-closed. Since $(X, \tau, \mathscr{I})$ is weak semi- $\mathscr{I}-R_{0},\{x\}$ is a $\Lambda_{I}^{s}$-set.

\section{Conclusion}

The notions of closed sets and some low separation axioms in topological spaces are extensively developed and used in many fields of applied sciences. The study of generalized closed sets and some low separation axioms have been found to be useful in computer science and digital topology. This paper is dealing with the concepts of $\mathscr{S}^{\star}$-closed sets and $\mathscr{S}^{\star}$-open sets which are defined by utilizing the notion of $\Lambda_{I^{-}}^{s}$ sets. Some properties of $\mathscr{S}^{\star}$-closed sets and $\mathscr{S}^{\star}$-open sets are obtained. Moreover, several interesting characterizations of semi- $\mathscr{I}-R_{0}$ ideal topological spaces are established. The ideas and results of this paper may motivate further research.

\section{References:}

[1] F.-G. Arenas, J. Dontchev and M. Ganster, On $\lambda$-closed sets and dual of generalized continuity, Questions Answers Gen. Topology 15, 1997, pp. 3-13.

[2] M. Caldas, J. Safari and G. Navalagi, More on $\lambda$ closed sets in topological spaces, Revista Colombiana de Matemáticas 41, 2007, pp. 355-369.

[3] M. Caldas and J. Safari, On some low separation axioms via $\lambda$-open and $\lambda$-closure operator, Rend. Circ. Mat. Palermo 54, 2005, pp. 195-208. 
[4] M. Caldas, S. Jafari and T. Noiri, Characterizations of $\Lambda_{\theta}-R_{0}$ and $\Lambda_{\theta}-R_{1}$ topological spaces, Acta Math. Hungar. 103, 2004, pp. 85-95.

[5] M. Caldas and S. Jafari, On some low separation axioms in topological spaces, Houston J. Math. 29, 2003, pp. 94-104.

[6] J. Dontchev, M. Ganster and T. Noiri, Unified operation approach of generalized closed sets via topological ideals, Math. Japon. 49, 1999, pp. 395-401.

[7] E. Ekici and T. Noiri, «-hyperconnected ideal topological spaces, Analele Stinn. Ale Univ. A. I. Cuza Din Iasi-Serie Noua-Mat. 58, 2012, pp. 120-129.

[8] E. Ekici, On $\gamma-U S$ spaces, Int. J. Pure Appl. Math. 25, 2005, pp. 163-172.

[9] E. Ekici, On $R$ spaces, Indian J. Math. 47, 2005, pp. 131-138.

[10] D.-N. Georgiuo, S. Jafari and T. Noiri, Properties of $(\Lambda, \delta)$-closed sets in topological spaces, Boll. Un. Mat. Ital. 7, 2004, pp. 745-756.

[11] E. Hatir, A. Keskin and T. Noiri, A note on strong $\beta-\mathscr{I}$-open sets and strongly $\beta-\mathscr{I}$ continuous functions, Acta Math. Hungar. 108, 2005, pp. 87-94.

[12] E. Hatir and T. Noiri, On semi- $\mathscr{I}$ - open sets and semi- $\mathscr{I}$-continuous functions, Acta Math. Hungar. 107, 2005, pp. 345-353.

[13] E. Hatir and T. Noiri, On decompositions of continuity via idealization, Acta Math. Hungar. 96, 2002, pp. 341-349.

[14] D.-S. Janković and T.-R. Hamlett, New topologies from old via ideals, Amer. Math. Monthly 97, 1990, pp. 295-310.

[15] A. Keskin and T. Noiri, On $\gamma-R_{0}$ and $\gamma-R_{1}$ spaces, Miskolc Math. Notes 10, 2009, pp. 137143.

[16] K. Kuratowski, Topology, Vol. I, AcademicPress, New York, 1966.

[17] H. Maki, Generalized $\Lambda$-closed sets and the associated closure operator, The Special Issue in Commemoration of Prof. Kazusada IKEDA' Retirement, 1986, pp. 139-146.

[18] J. Sanabria, E. Rosas and C. Carpintero, On $\Lambda_{I^{-}}^{\text {- }}$ sets and the related notions in ideal topological spaces, Math. Slovaca 63, 2013, pp. 1403-1411.

[19] N.-V. Veličko, $H$-closed topological spaces, Amer. Math. Soc. Transl. 78, 1968, pp. 102-118.

\section{Creative Commons Attribution License 4.0 (Attribution 4.0 International, CC BY 4.0)}

This article is published under the terms of the Creative Commons Attribution License 4.0 https://creativecommons.org/licenses/by/4.0/deed.en_US 